\title{
Morphological Aspects of the Myocarditis and Myositis in Calomys callosus Experimentally Infected with Trypanosoma cruzi: Fibrogenesis and Spontaneous Regression of Fibrosis
}

\author{
Sonia G Andrade ${ }^{+}$, Judith K Kloetzel", Monamaris M Borges", Victor J \\ Ferrans
}

\begin{abstract}
Centro de Pesquisas Gonçalo Moniz-FIOCRUZ/UFBA, Rua Valdemar Falcāo-121, 40295-001 Salvador, BA, Brasil "Instituto de Medicina Tropical de São Paulo and Departamento de Parasitologia, ICB/USP, 05403-000 São Paulo, SP, Brasil "'Pathology Branch National Heart, Blood and Lung Institute, NIH, Bethesda, MD 20892, USA
\end{abstract}

Calomys callosus a wild rodent, is a natural host of Trypanosoma cruzi. Twelve C. callosus were infected with $10^{5}$ trypomastigotes of the F strain (a myotropic strain) of T. cruzi. Parasitemia decreased on the 21st day becoming negative around the 40th day of infection. All animals survived but had positive parasitological tests, until the end of the experiment. The infected animals developed severe inflammation in the myocardium and skeletal muscle. This process was pronounced from the 26 th to the 30th day and gradually subsided from the 50th day becoming absent or residual on the 64th day after infection. Collagen was identified by the picro Sirius red method Fibrogenesis developed early, but regression of fibrosis occurred between the 50th and 64th day. Ultrastructural study disclosed a predominance of macrophages and fibroblasts in the inflammatory infiltrates, with small numbers of lymphocytes. Macrophages had active phagocytosis and showed points of contact with altered muscle cells. Different degrees of matrix expansion were present, with granular and fibrillar deposits and collagen bundles. These alterations subsided by the 64th days. Macrophages seem to be the main immune effector cell in the $\mathrm{C}$. callosus model of infection with $\mathrm{T}$. cruzi. The mechanisms involved in the rapid fibrogenesis and its regression deserve further investigation.

Key words: Trypanosoma cruzi - Calomys callosus - myocarditis - myositis - fibrogenesis - fibroclasia ultrastructure - collagen

Calomys callosus (Rodentia - Cricetidae), a natural reservoir of Trypanosoma cruzi, was first identified as harboring this parasite by Ribeiro (1973) and Mello and Teixeira (1977). The biology of C. callosus and its breeding in the laboratory have been described by Mello (1984). Since its identification as a natural reservoir, it has been used as a model for experimental $T$. cruzi infection (Mello et al. 1979, Mello \& Borges 1981, Borges et al. 1982). Recently, a comparative study was made of the evolution of the infection induced by different strains of $T$. cruzi in this model and in mice, including the correlation between activation of peritoneal macrophages and the histopathological findings (Borges et al. 1992). The F strain of $T$. cruzi evoked early macrophage infiltration and fibroblastic

Supported by CNPq.

+ Corresponding author

Received 22 December 1993

Accepted 20 April 1994 proliferation in myocardium and skeletal muscle, with conspicuous fibrogenesis. This process subsided spontaneously, without the development of chronic lesions such as those seen in mice (Andrade \& Grimaud 1986). Since myocarditis and myositis in Calomys showed peculiar features, with marked fibrogenesis followed by fibroclasia, the cellular changes and the responses of the extracellular matrix at different stages were investigated by light and electron microscopy in an attempt to clarify the pathogenesis of $T$. cruzi infection in this model.

\section{MATERIALS AND METHODS}

Twenty $C$. callosus raised in the Instituto de Medicina Tropical de Sāo Paulo, Brazil, were used in the study. Twelve were infected with the F strain of $T$. cruzi and eight were maintained as uninfected controls. Inocylations were performed intraperitoneally with $10^{5}$ trypomastigotes (blood forms) obtained from infected mice.

The F strain was originally obtained from PA D'Alessandro (Deane \& Kloetzel 1974) and has been maintained at the Instituto de Medicina 
Tropical de São Paulo, both in mice and in $C$. callosus. It is a myotropic strain, as previously shown by Borges et al. (1992). Parasitemia was evaluated daily by microscopic examination of peripheral blood under coverslips ( $22 \times 22 \mathrm{~mm})$, counting the numbers of parasites in $50 \mathrm{high}$ power (X400) fields. In animals in which direct blood examination was negative, blood subinoculation into newborn mice and xenodiagnosis were performed. For subinoculation, $0.1 \mathrm{ml}$ of citrated blood was injected intraperitoneally. Xenodiagnosis was performed using five 3 to 5 stage nymphs of Rhodnius prolixus. Infected and control animals were killed at 21,26,30,50 and 64 days after infection.

For histopathological study, samples of myocardium and skeletal muscle were formalinfixed, paraffin-embedded, and $5 \mu \mathrm{m}$-thick sections were stained with hematoxylin and eosin. Sections were also stained for collagen with the picro Sirius red method of Junqueira et al. (1979), using Rot-Sirius 3 (Chroma Gessellschaft) in a saturated aqueous solution of picric acid and Fast green as a counterstain. Sections were examined with bright field and polarized light optics using a Zeiss photo microscope II.

For ultrastructural study, small blocks of right atrium and skeletal muscle from the thigh were fixed in $2 \%$ glutaraldehyde in $0.1 \mathrm{M}$ cacodylate buffer, pH 7.4, for $2 \mathrm{hr}$ and postfixed with $1 \%$ Osmium tetroxide in $0.15 \mathrm{M}$ cacodylate buffer. After dehydration with ethanol the tissues were embedded in Poly-bed 812 resin. $1 \mu$ m-thick sections stained with Toluidine blue were examined by optical microscopy to select areas for electron microscopic study. Ultrathin sections were contrasted with uranyl acetate and lead citrate and examined with a JEOL 1200EX Electron Microscope at an accelerating voltage of $60 \mathrm{kV}$.

\section{RESULTS}

Infected C. callosus developed parasitemia, which peaked at the 21 st day of infection. Then it declined, becoming negative from the 40th day until the end of the experiment (64 days). Xenodiagnosis and/or subinoculation were positive in all the animals.

Histologic study - Until the 21 st day post infection parasitism was scarce in cardiac and skeletal muscle and was not detected in macrophages. From the 26th to the 30th day post infection, myocardial inflammatory lesions varied from mild to very severe, with few intracellular parasites. In one animal, diffuse necrosis of cardiac myocytes and marked inflammatory infiltration (mainly of macrophages and fibroblasts and some lymphocytes), were present together with abundant deposit of eosinophilic material in the interstitial connective tissue matrix (Figs 1a, b). From the 50th through the 64th day inflammatory lesions were reduced to focal mononuclear infiltrates and scarce interstitial matrix deposits. Inflammatory and degenerative lesions in skeletal muscle were severe from the 26th to the 30th day, with numerous intracellular parasites (amastigotes) forming large clusters. Marked infiltration of mononuclear cells was seen in the perivascular spaces, in the interstitium and around necrotic cells, sometimes in association with neutrophils, disintegrated parasites and cell debris (Fig. 2a). Macrophages and fibroblasts predominated in the infiltrate, with some small lymphocytes and plasmocytes and loose matrix deposits. Interstitial lesions formed by epithelioid cells were also seen (Fig. 2b). From the 50th to the 64th day, focal areas of muscle cells necrosis and mononuclear cell infiltration were seen. From the 50th to the 64th day, the interstitial spaces in skeletal muscle were free of inflammation and abnormal matrix deposits.

Staining for collagen - Examination of sections stained with picro Sirius red by direct and polarized light microscopy was used to identify fibrillar collagen. In the heart, small deposits of collagen were seen in the interstitium and in perivascular spaces, corresponding to focal inflammatory infiltrates. In the areas of severe inflammatory lesions, Sirius red-positive interstitial matrix deposits were abundant (Figs 4a, b). Occasionally, dense bundles of collagen extended from the subepicardium into the subjacent myocardium, in association with perivascular inflammatory infiltrates. In skeletal muscle, deposits with positive staining and birefringence were seen in the perivascular spaces and extended into other interstitial areas, forming long strands of collagen that delimited groups of muscle cells (Fig. 5c). On the 50th day, deposits of collagen were limited to the perivascular spaces and focal areas of the interstitium. By the 64th day, only small focal perivascular collagen deposits were present.

Sections of the heart and skeletal muscles from uninfected control animals showed essentially normal structure. Fine strands of collagen were seen as isolated bundles in the epicardium and in the perivascular and interstitial spaces of skeletal muscle (Figs 5a, b).

Ultrastructural study - Heart: from the 24th to the 30th day of infection sections of right atrium showed a predominance of macrophages, with abundant lysosomes, in the inflammatory infiltrates. These lysosomes contained electrondense materials that sometimes were identified as parasite debris by the presence of submembranous microtubules (Fig. 6). Points of contact between cytoplasmic processes of macrophages and the basement membranes of myocytes were seen (Fig. 6). Fibroblasts showing prominent cisterns of endoplasmic reticulum 

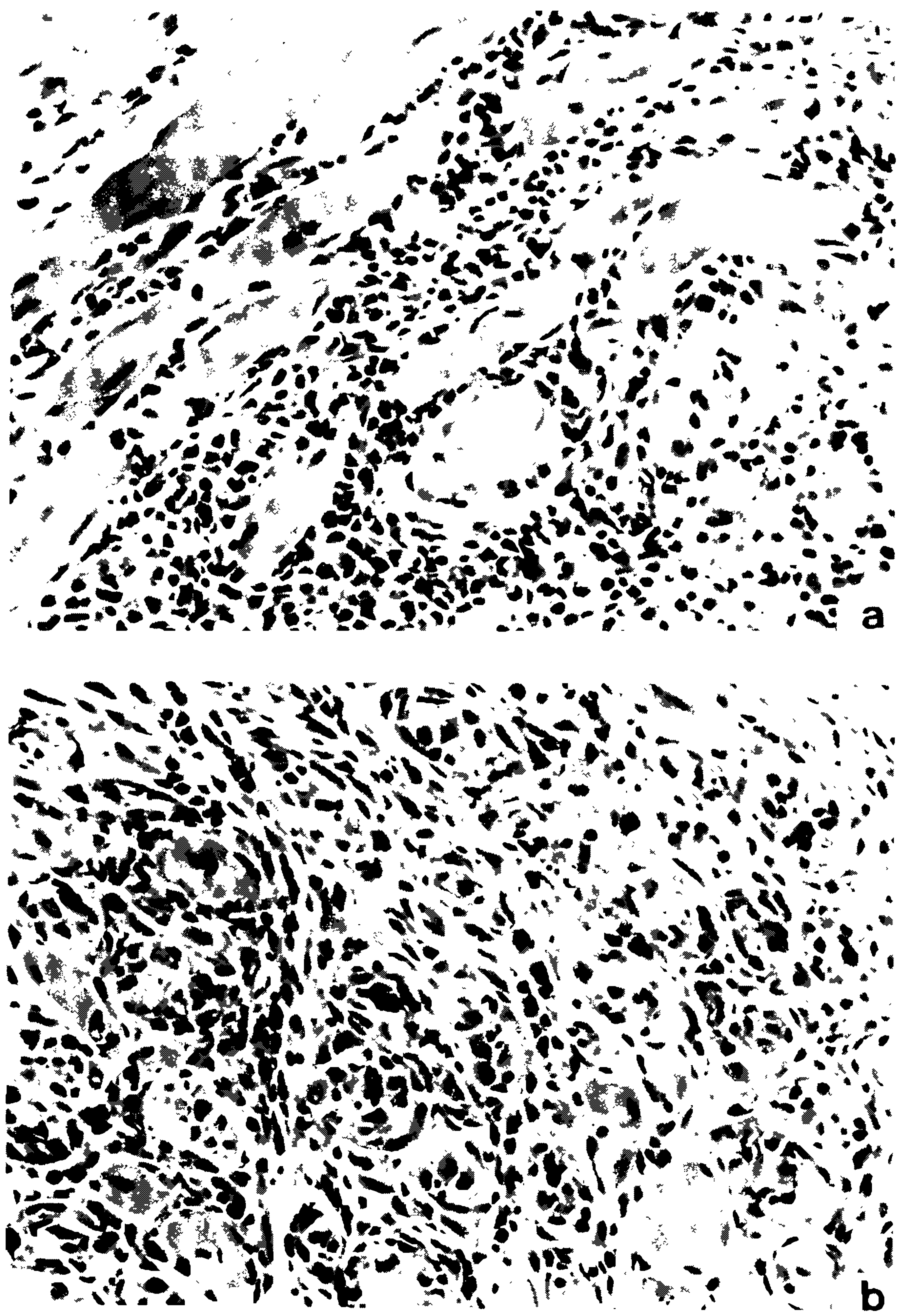

Fig. 1 : myocardium - a: intense difuse myocarditis with necrosis of cardiac cells and mononuclear cells infiltration and deposit of cosinophilic material in the interstitium, 26th day of infection; b: cardiac cells are mostly destroyed and replaced by macrophages infiltration and fibroblasts proliferation, 30th day of infection. $400 \mathrm{X}$.

were present in small numbers by the 26 th through the 30th day of infection. Lymphocytes were present only in small numbers and were mostly of the small, agranular type. Myocardial capillaries appeared normal. Abundant matrix deposits formed by granular and fibrillar material and dense collagen bundles were seen (Fig. 7). The collagen deposits in subepicardial areas were more prominent and were adnixed with elastic fibers.

Myocytes showed focal alterations such as disruption of $\mathrm{Z}$ bands, mitochondrial vacuoliza- 

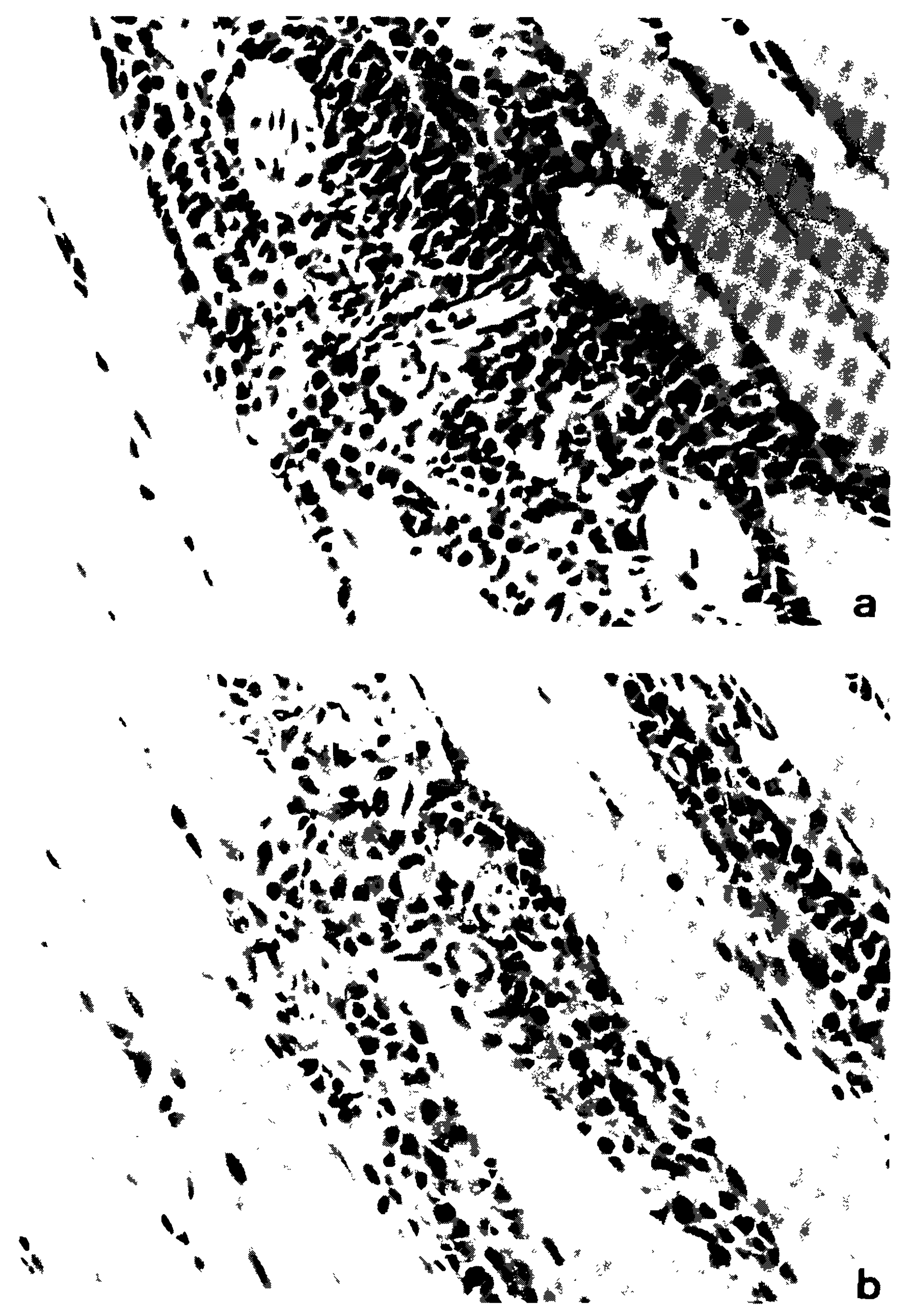

Fig. 2 : skeletal muscle - s: focal destruction of muscle cells and intense interstitial mononuclear infiltration, 26th day of infection; $b$ : interstitial infiltration with mononuclear cells predominantly epithelioid cells, 30th day of infection.400X.

tion, formation of intramitochondrial amorphous densities and myofibrillar disorganization (Fig. 8). These changes were associated with the presence of focal inflammatory infiltrates. By the 50th day, macrophages and fibroblasts were less numerous. Interstitial spaces contained amorphous or finely fibrillar material associated with isolated collagen fibrills which showed indistinct periodicity and a marked increase in electron density (Fig. 9). By the 64th day of infection, small focal inflammatory infiltrates were seen in association with altered myocytes.

Skeletal muscle - From the 26th to the 30th day after infection, ultrastructural alterations 

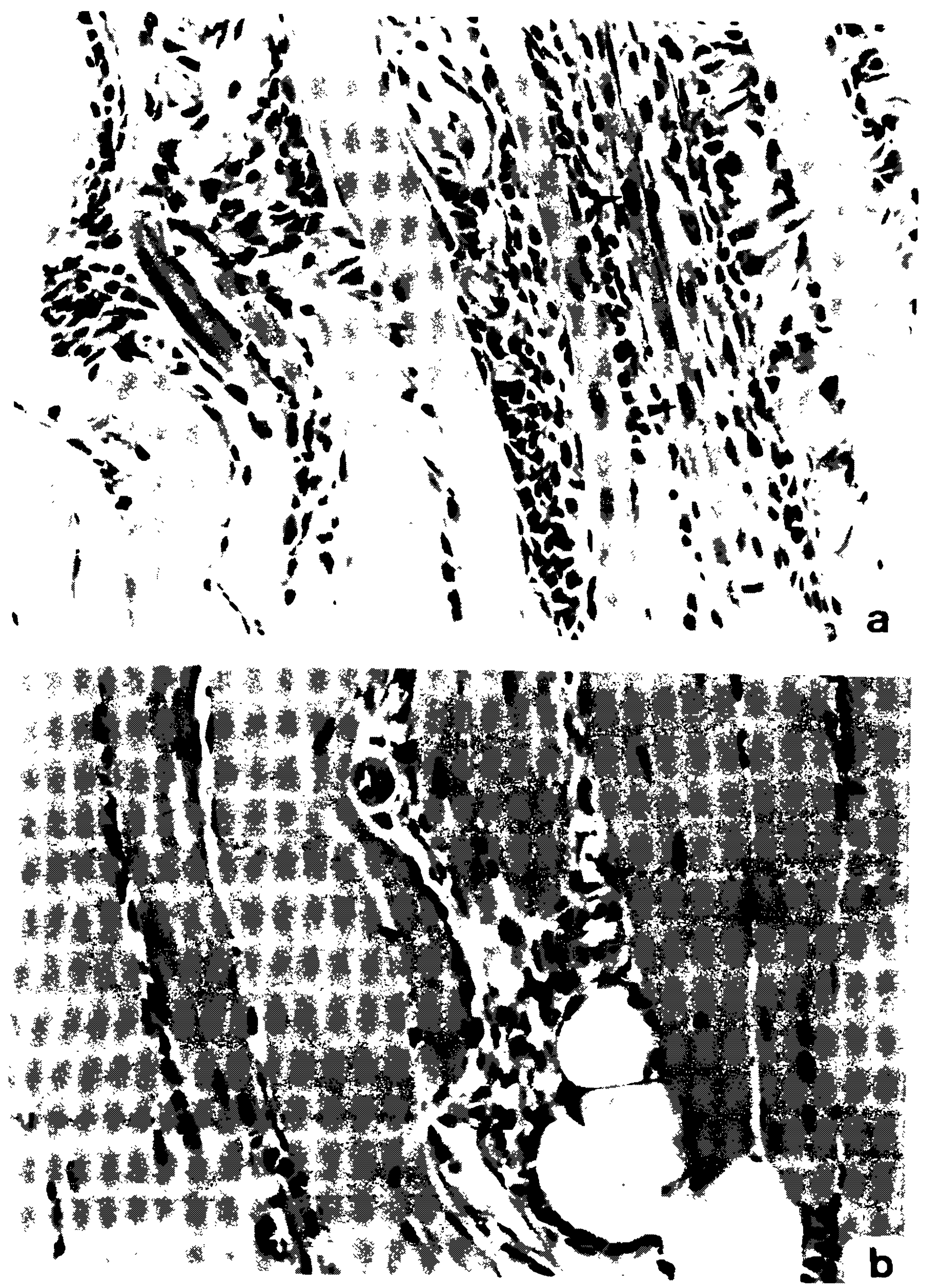

Fig. 3 : skeletal muscle, regressive lesions - a : interstitium with perivascular mononuclear infiltration with low cellularity; area of muscle destruction and sarcolemal proliferation (arrow), 56 th day of infection; $b$ : residual perivascular inflammatory lesion with adipose infiltration, 64 th day of infection. $400 \mathrm{X}$.

were more severe in skeletal muscle than in cardiac muscle. Inflammatory infiltrate consisted mainly of macrophages with abundant lysosomes and phagosomes, fibroblasts and a few lymphocytes. Macrophages with epithelioid differentiation were found in the interstitial spaces (Fig. 10). In some animals, the predominance of fibroblasts in the interstitium was remarkable, and these were almost the only type of cell present in such areas. Extracellular matrix deposits were abundant and consisted of fibrillar and granular material and collagen bundles (Fig. 11). Disintegrated parasites were rarely seen within the cytoplasm of altered muscle cells. By 


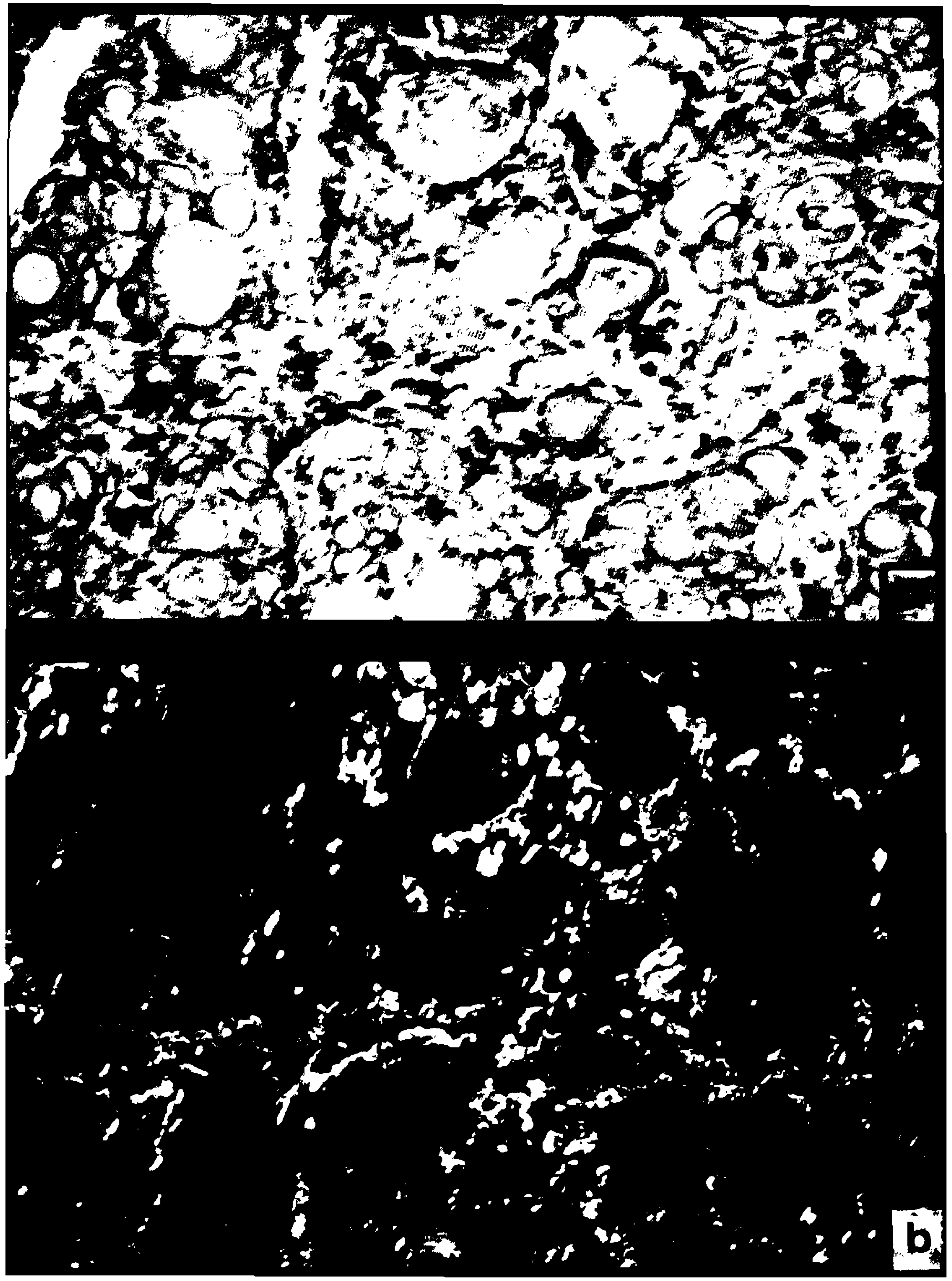

Fig. 4 : myocardium, red Sinius stained secctions - a: area of intense cardiac cells destruction with enlargement of the interstitial spaces and irregular deposits of collagen; $b$ : the same area under polarized light, showing specific bi-refringence of the collagen bundles, 30 days. $400 \mathrm{X}$.

the 50th and the 64th day, a decrease in the numbers of macrophages and fibroblasts was detected. Interstitial matrix was scarce, with few collagen bundies. Focal cellular infiltrates were seen and these correlated with the presence of necrosis of muscle cells in contact with macrophage processes (Fig. 12).

Ultrathin sections of myocardium and skeletal muscle of uninfected control animals showed normal structure. 

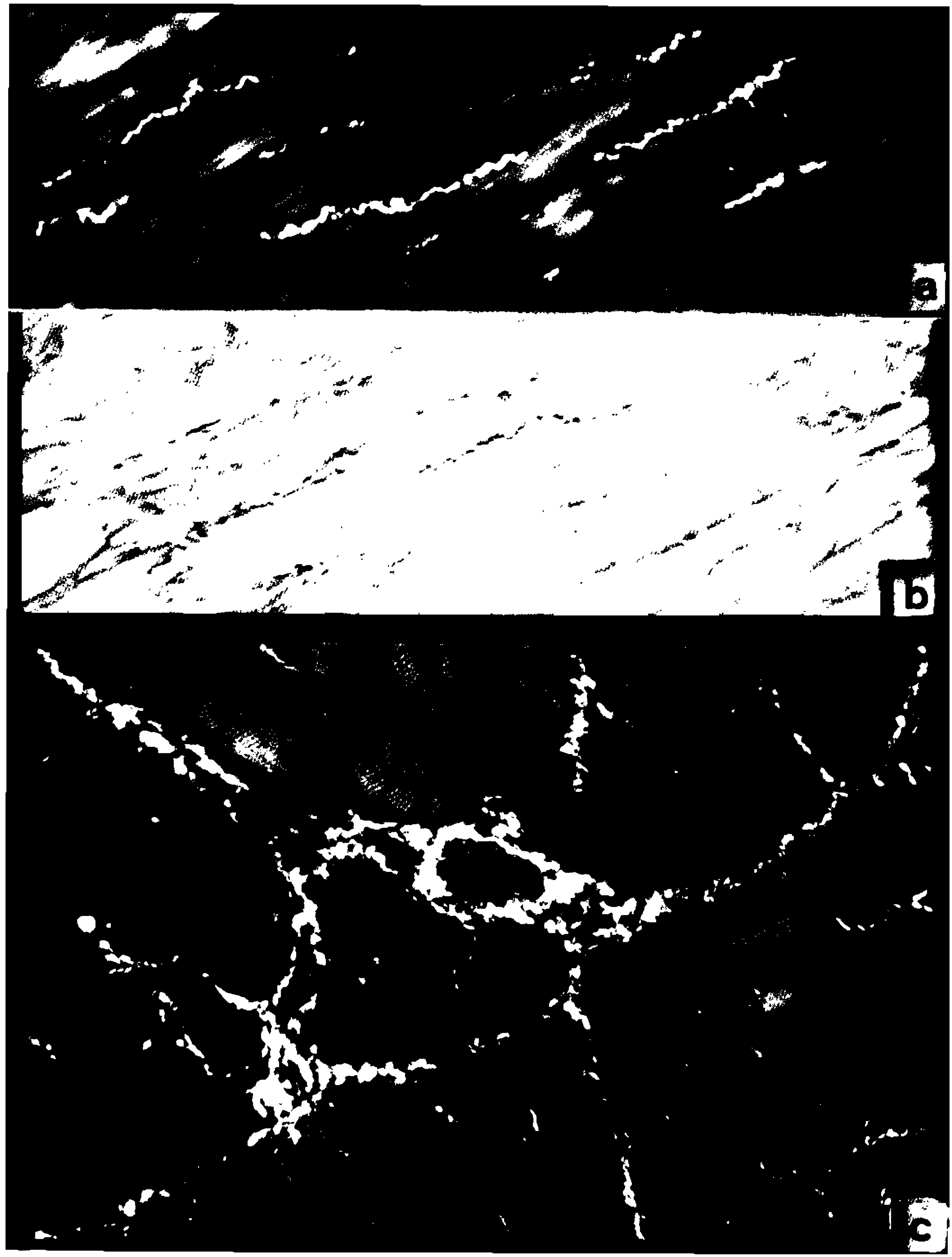

Fig 5 : skeletal muscle, staining with Red Sirius - $a$ and b: control uniinfected animal showing fine strands of normal collagen deposits in the interstitium viewed under polarized (a) or direct (b) light; $c$ - interstitial deposits of collagen 30 days after infection (polarized light). 400X.

\section{DISCUSSION}

A peculiar evolution of $T$. cruzi (F strain) infection in C.callosus as compared with that in the mouse, has been previously described (Borges et al.1992). C. callosus was more resistant and sur- vived the infection, while a high mortality was observed in the infected mice in the acute phase. The present investigation shows that, although being "resistant" in the sense of having a low mortality, C.callosus developed severe lesions in 


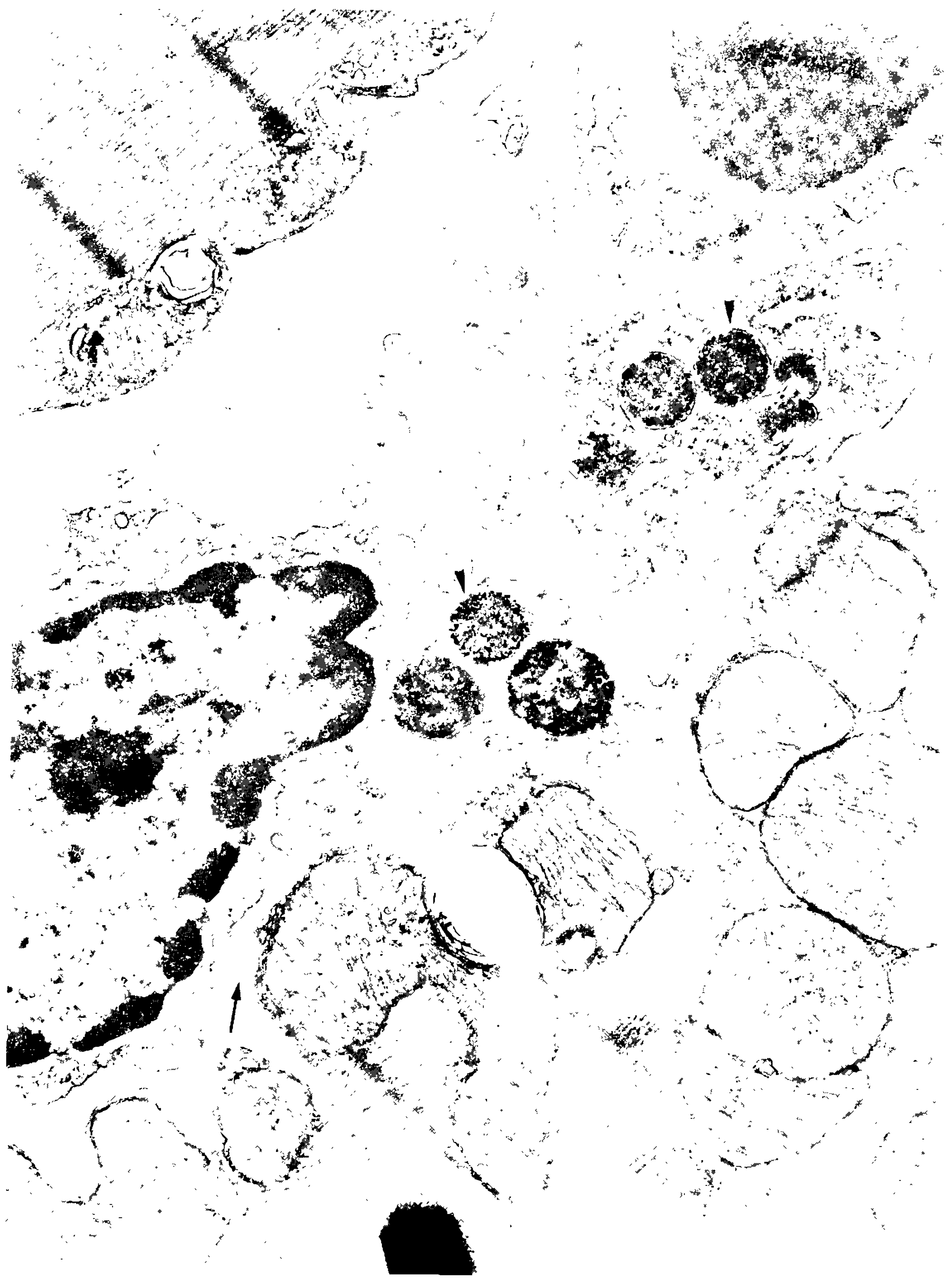

Fig.6 : myocardium - widened interstitial space in the myocardium (26th day). Macrophage contains degenerate parasites with conspicuous microtubules within phagosomes (short arrows). Macrophage processes make contact with the basement membrane of a cardiac myocyte (long arrow). 10,000X 


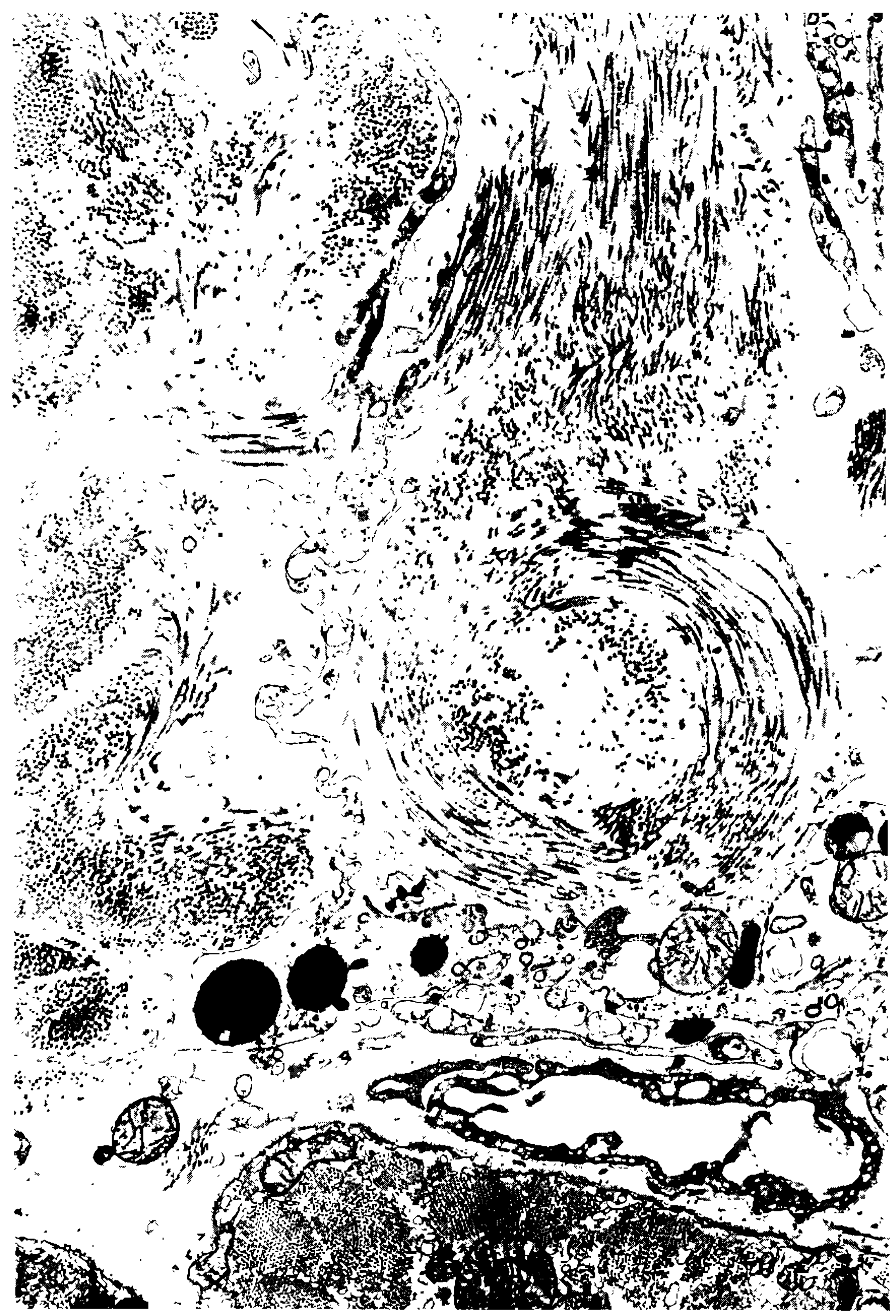

Fig. 7 : myocardium - widened interstitial space in the myocardium contains abundant collagen deposits forming discrete bundles. A blood capillary shows normal structure (30th day). 6,000X.

skeletal muscle and myocardium. This infection was associated with a severe fibrogenic process, mainly in skeletal muscle, since the early phase ( 26 to 30 days). From the 50 th day of infection regression of the lesions was observed, and inflammatory and fibrotic processes were focal and residual by the 64 th day. Although direct parasitemia was negative, this did not indicate eradication of the infection, since parasitological tests still yielded positive results. Survival of all the animals throughout the experiment indicated that $C$. callosus controls the infection and develops a host-parasite equilibrium, an ideal condition for becoming a reservoir of parasites. The strain of $T$. cruzi is important in determining the severity of the histopathological lesions in $C$. callosus. As previously shown (Borges et al. 1992), the F strain induced severe lesions up to a late phase after infection; however, the lesions induced by the $Y$ strain subsided entirely 20 days 


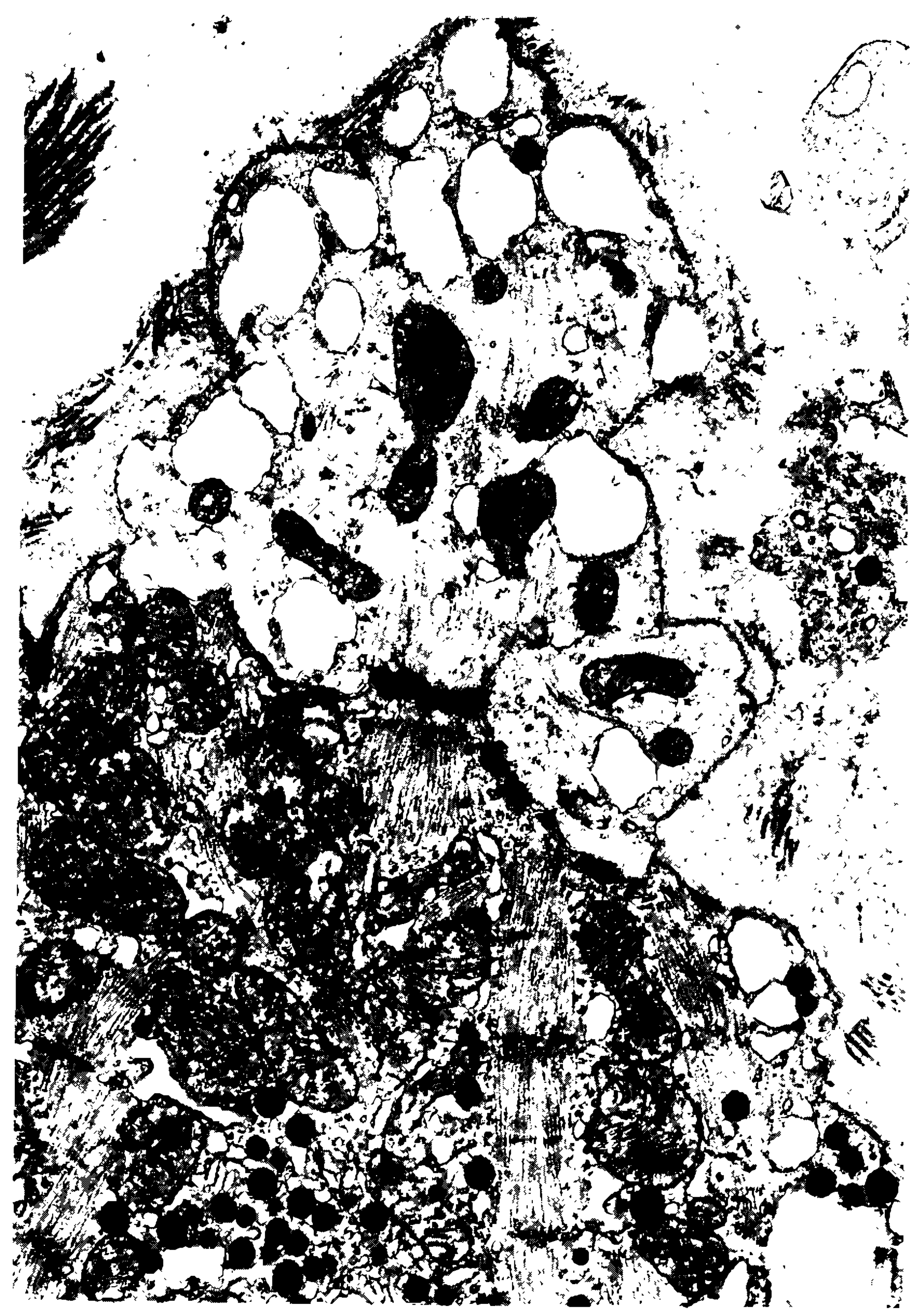

Fig. 8: myocardium - portions of two adjacente myocytes. One of these contains many mitocondria and specific atrial granules; the other cell shows myofibrillar loss and dilatation of sarcoplasmic reticulum $(30$ th day). 8,000 X.

after infection. The present ultrastructural study disclosed details of the progressive and spontaneously regressive processes that occurred during the infection of $C$. callosus with the $F$ strain of $T$. cruzi. Macrophages were the most prominent cells in the inflammatory infiltrate and appear to be responsible for the clearance of parasites from infected tissues. These cells showed morphological signs of activation, as indicated by long cytoplasmic processes and abundant lysosomes, which often contained phagocytosed, degenerating parasites. These findings suggest that parasite clearance and control of the infection are dependent upon activation of the macrophages. Contacts between cytoplasmic processes of macrophages and the basement membranes of cardiac myocytes constituted evidence of direct interactions between these two types of cells. A damaging effect of macrophages on the membranes of cardiac myocytes has been previously described in the mouse model (Rossi 1990). Macrophages appear 


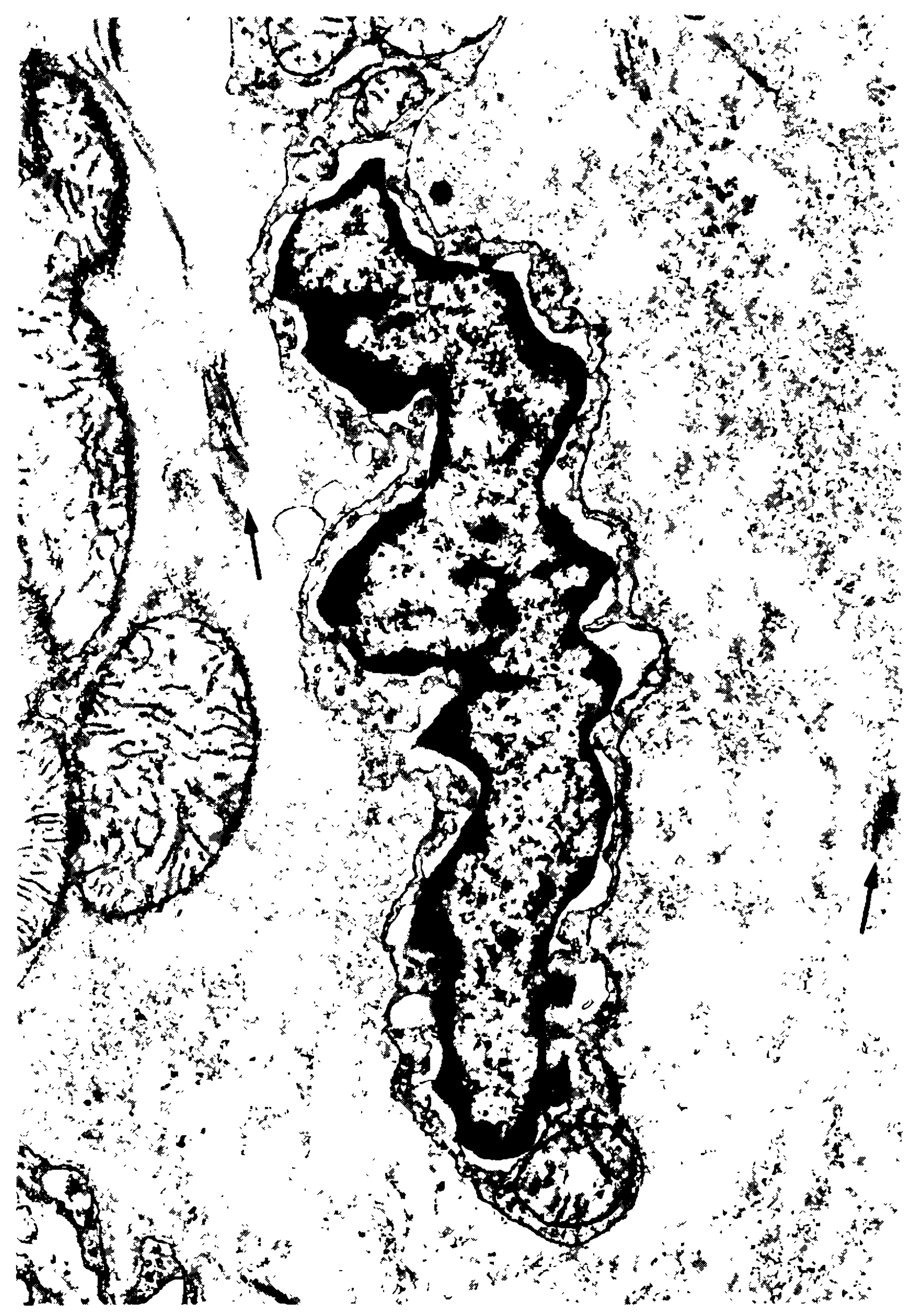

Fig 9 : myocardium - enlarged interstitial space of the myocardium (50th day) with fibroblast and scanty matnx deposit and few collagen bundles with increased electrondensity (arrows), 6,000X.

to be the main immune effector cells in the Calomys model of infection with $T$. cruzi. Activation and proliferation of fibroblasts probably were induced by the release of fibrogenic cytokines by the macrophages involved in the inflammatory process. Delayed type hypersensitivity has been shown by several authors to be involved in chronic infection with $T$. cruzi (Santos-Buch \& Teixeira 1974, Ribeiro dos Santos et al. 1991, Andrade 1991). It is possible that a significant immunosuppression of delayed type hypersensitivity, as described in mice (Scott 1981) and in dogs (Andrade et al. 1987), is involved in the rapid regression of the inflammatory lesions in the $C$. callosus model. The reversibility of collagen deposition in chronically infected mice, under curative chemotherapy, has been previously shown (Andrade et al, 1991). Although morphological evidence of fibroclasia was present in C. callosus, subsiding fibrosis seemed to be more dependent on decreased in fibrogenesis than 


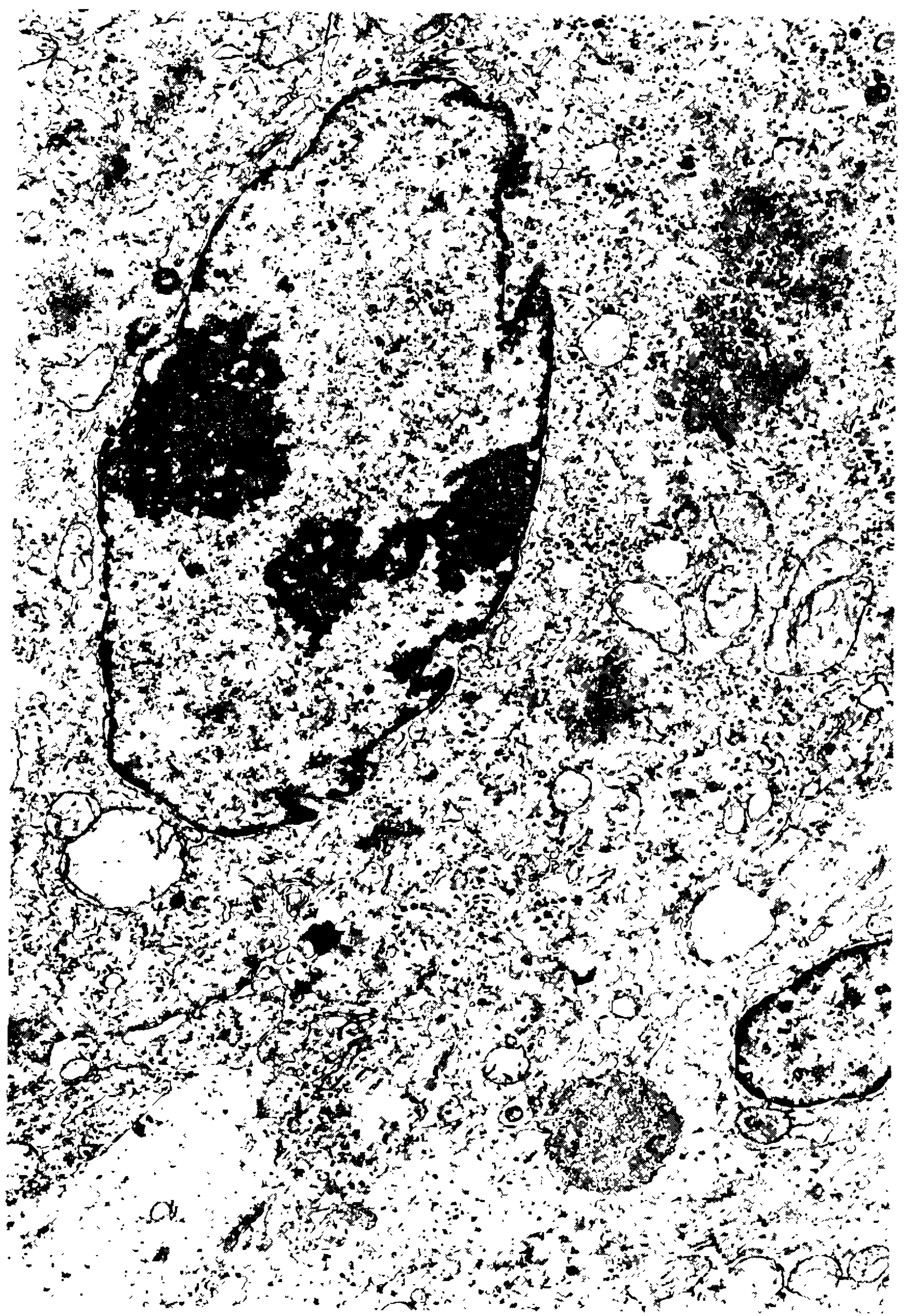

Fig. 10 : skeletal muscle - epithelioid cell is present in a granulomatous reaction in the interstitial space of skeletal muscle and show abundant cytoplasm in which numerous ribosomes, and few mitochondria are found (30th day). I ysosomes are not present. One nucleus contains a thin rim of peripherically located chromatin and two nucleoli. $8,000 \mathrm{X}$

was the case in mice. A down regulation of fibrogenesis, mediated by suppression of delayed type hypersensitivity, may be involved in the reduction in collagen. A similar mechanism has been described in schistosomiasis (Wyler et al. 1992). Fibrogenesis and spontaneous regression of fibrosis in this model deserve further investigations. Fibrosis is one of the most important features of chronic Chagas' cardionyopathy and shows a progressive evolution in the mouse model (Andrade et al. 1989). According to Weber et al. (1993), fibrillar collagen deposits in the myocardial interstitium are of paramount importance in the development of cardiac failure, and particularly of diastolic dysfunction, in different cardiac diseases. These observations on the highly unusual features of $T$. cruzi infection in $C$. callosus, suggest that this species may become an useful animal model for the study of myocardial inflammation and fibrosis. 


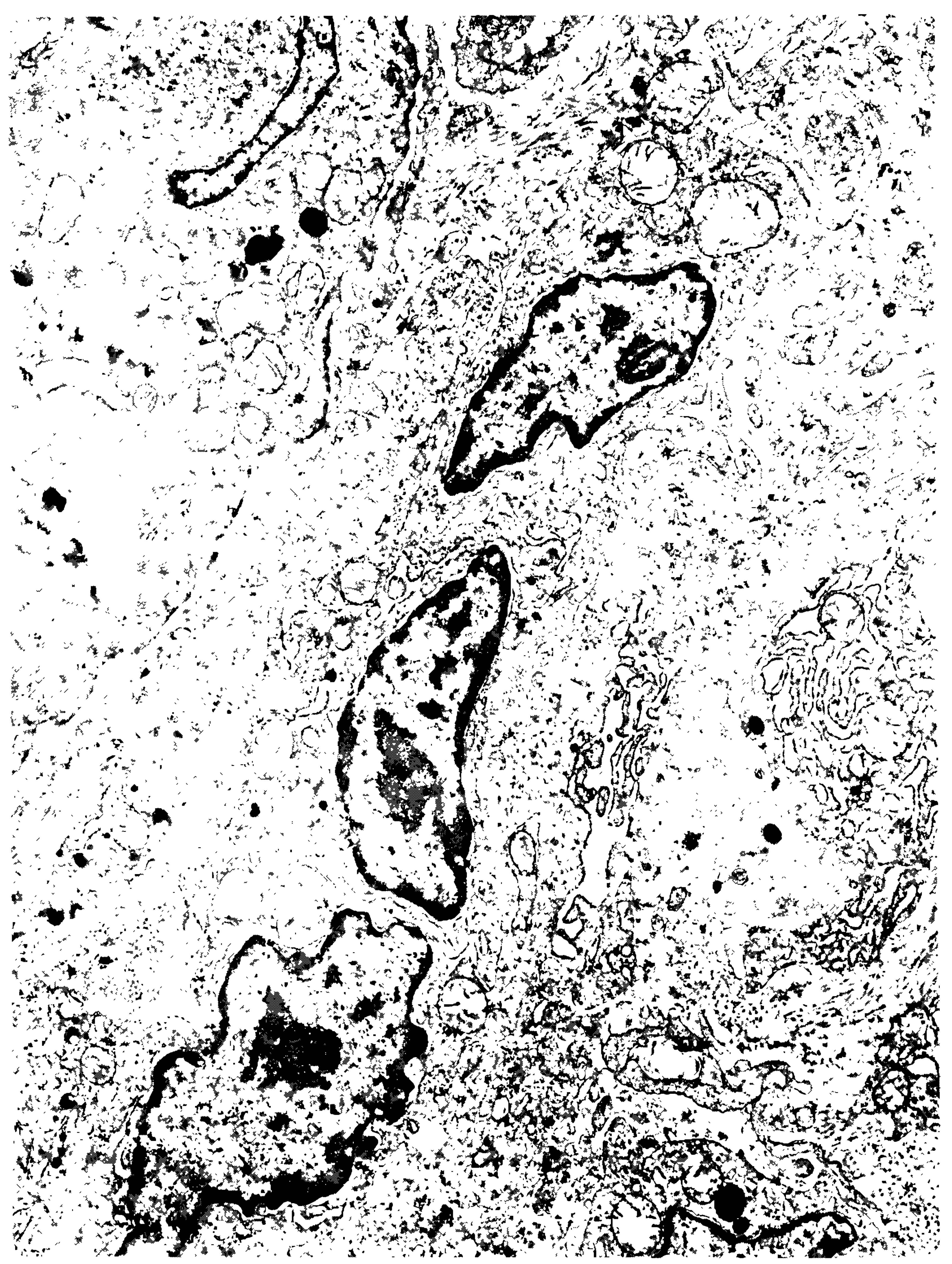

Fig. 11 : skeletal muscle - enlarged interstitial space of skeletal muscle showing several fibroblasts with prominent endoplasmic reticulum, cell processes and granular and fibrillar matrix deposits (30th day). The fibroblasts are the only type of cell present in this area. 8,000X. 


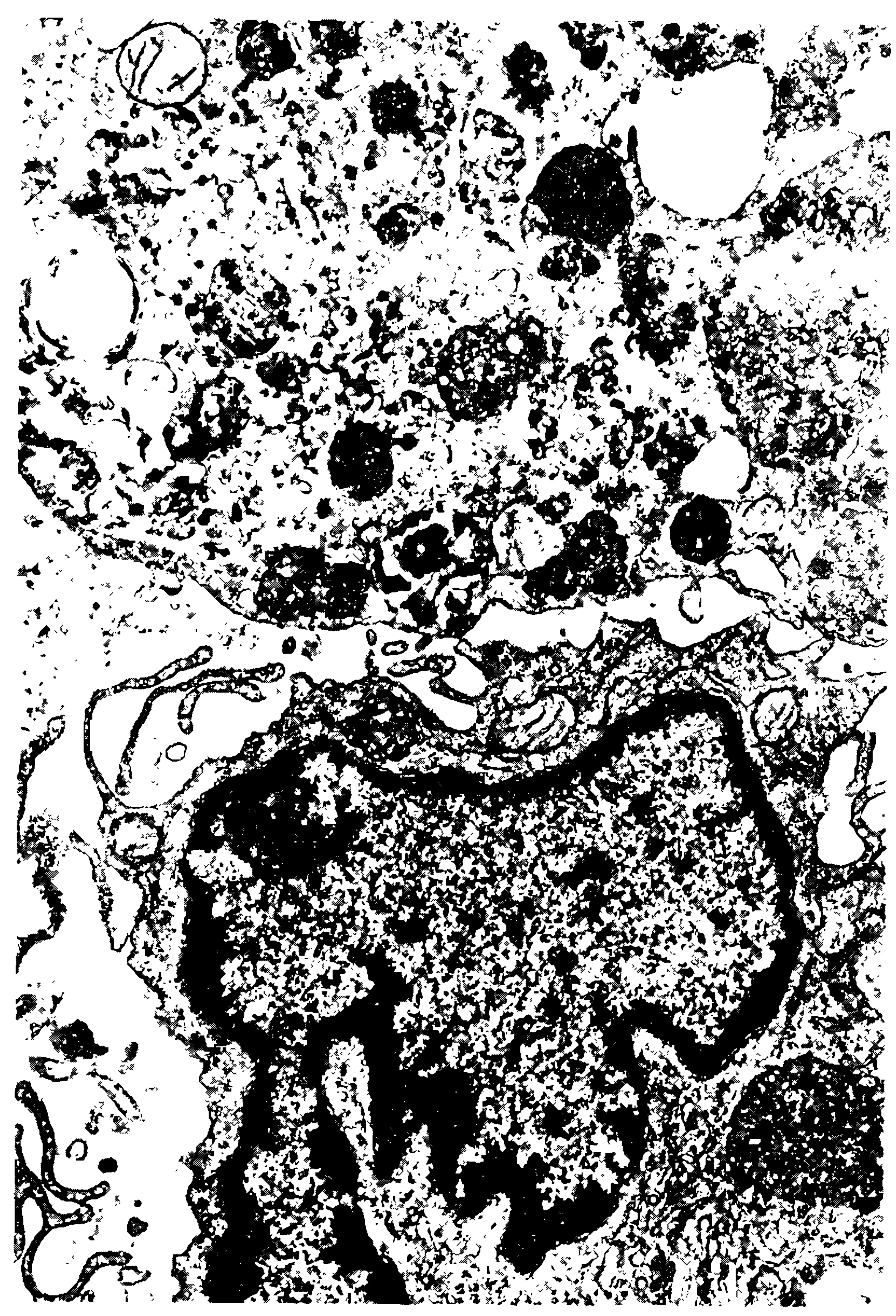

Fig 12 : skeletal muscle - necrotic skeletal muscle cell in an area of focal residual damage shows focal cytoplasmic disintegration, myofibrillar loss, lysosomes and mitochondrial, condensation (50th day). Cytoplasmic processes of a macrophage are in contact with the myocyte. $10,000 \mathrm{X}$.

\section{REFERENCES}

Andrade SG, Grimaud JA 1986. Chronic murine myocarditis due to Trypanosoma cruzi:an ultrastructural study and immunocytochemical characterization of cardiac interstitial matrix. Mem Inst Oswaldo Cruz 81:29-41.

Andrade SG, Grimaud JA, Guerret S 1989. Sequential changes of the connective matrix components of the myocardium (fibronectin and laminin) and evolution of cardiac fibrosis in mice infected with Trypanosoma cruzi. Ain J Trop Med Hyg 40:250 260.

Andrade SG, Stocker-Guerret S, Pimentel AS, Grimaud JA 1991. Reversibility of candiac fibrosis in mice chronically infected with Trypanosoma cruzi under chemothemapy.Mem Inst Osualdo Cruz 86: 187-200.

Andrade ZA, Andrade SG, Sadigursky M 1987. Enhancement of chronic Trypanosoma cruzi myocarditis in dogs treated with low doses of Cyclophosphamide. Amer J Path 127:467-473.

Andrade ZA 1991. Pathogenesis of Chagas'disease. Res Immunol 142:126-129.

Borges MM, Andrade SG, Pilatti CG, Prado Jr. JC, Kloetzel JK 1992. Macrophage activation and histopathological findings in Calomys collosus and Swiss mice infected with several strains of Trypanosoma cruzi. Mem Inst Oswaldo Cruz 87:493-502.

Borges MM, Mello DA, Teixeira ML 1982. Infecçāo experimental do $C$. callosus (Rodentia/cricetidae) 
com Trypanosoma cruzi. Rev Saúde Públ (São Paulo).16:233-242.

Deane MP, Kloetzel JK. 1974. Lack of protection against Trypanosoma cruzi by multiple doses of $T$. lewisi culture forms. A discussion on some strains of "lewisi". Exp Parasitol 35:406-410.

Junqueirs LCU, Bignolas G, Brentani RR 1979. Picrosirius staining plus polarization microscopy, a specific method for collagen detection in tissue sections. Histochem $J$ 11:447-455.

Mello DA 1984. Calomys callosus, Renger, 1830 (Rodentia-Cricetidae): sua caracterizaçào, distribuição, biologia, criação e manejo de uma cepa em laboratorio. Mem Inst Oswaldo Cruz 76: 61-69.

Mello DA, Borges MM 1981. Primeiro encontro do Triatoma costalimai naturalmente infectado pelo Trypanosoma cruzi: estudo de aspéctos biológicos da amostra isolada. Mem Inst Oswaldo Cruz 76: 6169.

Mello DA, Valin E, Teixeira ML 1979. Alguns aspectos do comportamento de cepas silvestres do Trypanosoma cruzi em camundongos e Calomys callosus (Rodentia). Rev Saude Publ (São Paulo) 13: 314 - 332 .
Mello DA, Teixeira ML 1977. Nota sobre a infecçāo natural do Calomys expulsus (Lund, 1841) (Cricetidae-Rodentia) pelo Trypanosoma cruzi. Rev Saúde Públ (São Paulo) 11:561- 564.

Ribeiro RD 1973 . Novos reservatorios do Trypanasoma cruzi Rev Bras Biol 3: 429 - 537.

Ribeiro dos Santos R, Pirmez C, Savino W 1991. Role of autoreactive immunological mechanisms in chagasic carditis. Res Immunol J42:134-137.

Rossi MA 1990. Myocardial damage in Trypanosoma. cruzi myocarditis: a role for macrophages. Can $J$ Card 6: 293-298.

Santos-Buch CA, Teixeira ARL 1974. The immunology of experimental Chagas disease.-III. Rejection of allogeneic heart cells in vitro. $J$ Exp Med 140:38. 53.

Scott MT 1981. Delayed hypersensitivity to Trypanosoma cruzi in mice: specific suppressor cells in chronic infection. Immunology 44: 409 417.

Weber KT, Brilla CG, Janicki JS 1993. Myocardial fibrosis: functional significance and regulatory factors. Cardiovasc Res 27: 341-348.

Wyler DJ 1992. Why does liv, fibrosis occur in schistosomiasis? Parasitol Today 8: 277 - 279. 